Portland State University

PDXScholar

6-16-2021

\title{
COVID-19 and Palliative Care Through the Provider Perspective
}

Michaelyn V. Truong

Portland State University

Follow this and additional works at: https://pdxscholar.library.pdx.edu/honorstheses

Part of the Quality Improvement Commons

Let us know how access to this document benefits you.

\section{Recommended Citation}

Truong, Michaelyn V., "COVID-19 and Palliative Care Through the Provider Perspective" (2021). University Honors Theses. Paper 1050.

https://doi.org/10.15760/honors.1076

This Thesis is brought to you for free and open access. It has been accepted for inclusion in University Honors Theses by an authorized administrator of PDXScholar. Please contact us if we can make this document more accessible: pdxscholar@pdx.edu. 


\title{
COVID-19 and Palliative Care Through the Provider Perspective
}

\author{
by \\ Michaelyn Truong \\ An undergraduate honors thesis submitted in partial fulfillment of the \\ requirements for the degree of \\ Bachelor of Science \\ in \\ University Honors \\ and \\ General Science \\ Thesis Adviser \\ Samantha Underwood \\ Portland State University




\begin{abstract}
The purpose of this study was to analyze the OHSU palliative care team response to the COVID-19 pandemic and the impact of modified pandemic operations on delivering care to patients. The study was conducted through interviews with members of the palliative care teams from the Oregon Health \& Sciences University and the Portland Veteran Affairs Medical Center. Questions were asked to understand the changes in care due to the pandemic and their overall perception of these changes. Responses were analyzed through the qualitative analysis software NVIVO12. The findings of this study showed the challenges with telemedicine were limitations with communications and adapting to using technology, while the benefits of telemedicine were increased flexibility in time and access to patients. The study demonstrated that despite the challenges involved with providing palliative care during the pandemic, telemedicine was a viable and safe option to care for patients. In certain cases, remote work even provided more flexibility in meeting with patients and increased accessibility for providers to have interdisciplinary discussions.
\end{abstract}

\title{
Introduction
}

Palliative care is a subspecialty of medicine that provides support to critically ill patients (What Are Palliative Care and Hospice Care?, 2021). In order to address all of the domains that make up a comprehensive palliative care assessment, a palliative care team is comprised of an interprofessional team. This interprofessional team includes specialty-trained physicians, physician assistants, nurse practitioners, psychologists, social workers and chaplains. In addition to addressing complex symptoms associated with serious illness, palliative care also aims to ensure the care patients receive aligns with their overall goals and wishes. (Crawford \& Price, 
2003). Palliative care helps reduce discomfort from aggressive treatments/procedures, provide psychological support to patients and families, and overall reduce hospital costs (Bernacki \& Block, 2014).

Much of palliative care is traditionally delivered in-person, either in the inpatient hospital setting or the outpatient clinic. Providers can address and discuss sensitive health issues with patients and families, as well as rely on facial cues and body language to guide the conversation. However, with the COVID-19 pandemic, social distancing restrictions have put a limit on contact between care providers and patients and families (Ting et al., 2020). A recent study shows that $50 \%$ of COVID-19 positive patients admitted to the ICU die and are highly likely to have palliative care needs (Lovell et al., 2020).

Currently, there are very few reports describing the efficacy of modified palliative care operations during the pandemic. This study will examine how the pandemic has affected the delivery of palliative care from the perspective of the interprofessional teams at Oregon Health and Science University (OHSU) and the Portland Veteran Affairs Medical Center (Portland VA or VA). The purpose of this study is to analyze the response of the palliative care teams at OHSU and the Portland VA to the COVID-19 pandemic and the impact of modified pandemic operations on delivering care to patients.

OHSU and the Portland VA are adjacently located hospitals in Portland, Oregon, and they each employ separate interprofessional palliative care teams. Together, OHSU and the Portland VA receive over 2 million patient visits yearly and serve patients from all of Oregon and Southwest Washington (OHSU Facts $\mid$ OHSU, n.d.) (US Department of Veterans Affairs, Veterans Health Administration, n.d.). 


\section{Methods}

Interviews were conducted with members of the palliative care team at OHSU as well as the Portland VA. Members were contacted via email introducing the project. After consenting to be interviewed, interviews were conducted virtually via WebEx or Zoom video conference, with the exception of one written answer response. Participants included 3 physicians, 1 psychologist, 1 nurse practitioner, 1 social worker, and 2 chaplains.

Participants were asked questions designed to understand how the palliative care team at their institution was prepared for the pandemic as well as how the pandemic affected the provision of palliative care.

Transcriptions of each interview were provided by the transcription software Otter or through WebEx. NVIVO 12 was used to code specific answers from the interviews, gathering these codes into themes that summarized care providers' experience during the pandemic.

\section{Results}

\section{Pandemic Preparation}

One of the greatest concerns for healthcare professionals during a pandemic is overwhelming the hospital system. At OHSU and the Portland VA, extensive effort was put into preventing the spread of the disease, which could increase the number of patients and incapacitate the healthcare workforce. At the end of March 2020, both interprofessional palliative care teams at OHSU and the Portland VA transitioned to providing telehealthcare, and many began working remotely from home. The timing of this transition coincided with the State of Oregon's Executive Order No. 20-12 (“Stay Home, Save Lives”). Some staff began returning 
to the hospital to provide face-to-face patient care later in 2020 - at the Portland VA in May and later in the summer at OHSU.

At the VA, the decision to transition care away from being in-person was made with input from representatives of the interprofessional team. Palliative medicine, psychology and social work trainees were among interprofessional team members who no longer came to the hospital.

The VA was reported to have a history of providing telehealth with its proprietary virtual platform, Veteran Video Connect (VVC). Telehealth deployment at the VA was described by many providers to be rapid and successful, with installation of the necessary hardware for providing virtual care completed swiftly. Providers noted that the VA was able to make phone and video care options available to all members of the team.

An OHSU physician noted that the decision to move to virtual care was to protect the small team of palliative care providers. At OHSU, the palliative care team combined forces with the geriatric patient service, which helped to nearly double the number of those able to provide palliative care services. The team also began rigorously triaging consults in order to prevent maxing out the availability of their finite resources. In order to create greater access to palliative services, the team's schedule was extended beyond the traditional pre-pandemic hours. One care provider was available by phone after hours on nights and weekends and was available to complete consults and join family meetings or goals of care discussions.

\section{Changes to Care}

The greatest change to the interprofessional palliative care team made during the pandemic was utilizing telemedicine to provide palliative services to patients. This was accomplished by providing care in the form of phone calls or video calls using various platforms 
set up by each institution. At OHSU and the VA, telemedicine was implemented for both inpatient and outpatient encounters.

While some members of the interprofessional team at the VA transitioned entirely to remote work, others occasionally saw patients in the hospital, using their discretion to see patients in-person on a case-by-case basis. Until restrictions relaxed later in 2020, most of the time these providers still ultimately opted for virtual care.

\section{Telemedicine}

Providing care virtually revealed many technological limitations that may have existed long before. For care providers who were not seeing patients in person, technological difficulties and accessibility issues presented barriers to virtual meetings, whether with their colleagues or with patients and families. For many providers, the transition from working in person to setting up the necessary technology and learning to use it were a steep learning curve. Care providers had to set up their remote platforms at home without direct assistance.

Telephone. Phone appointments became the default method for most care providers. A nurse practitioner noted that about $80 \%$ of her contact with patients in her outpatient clinic was conducted via phone. For some providers, phone calls were easier to schedule, allowed for more flexibility, and required less coordination to complete.

Patients were noted by providers to prefer phone visits because of greater comfort levels with telephone technology. Some patients' physical limitations because of their illness made it difficult to hold the larger video devices for extended periods of time. Some providers also felt their patients were more open with their responses when conducting visits via telephone. 
Chaplains at OHSU did not have access to work-provided video conferencing technology and relied on work cell phones to communicate with patients. The chaplains felt phone visits provided ease of communication with their patients.

Despite being the default method for most providers, phone appointments also presented certain challenges. Even when providing care over the telephone, as many as 7 out of the 9 care providers interviewed expressed that phone calls were not ideal for communication, in both quantity and quality. Some felt symptom assessment was hindered by not being able to see patients and others noted that nonverbal communication was strained, making rapport-building more challenging. Many patients missed their outpatient phone appointments. One care provider reported it was especially challenging when patients missed their appointments because they worried about the potential for those patients' symptoms to worsen. Because of this, they spent more time than usual coordinating follow-up phone calls for those patients.

Whereas some providers felt phone calls were easier to schedule, others thought the necessary coordination was a barrier to efficiency. Despite the fewer number of steps needed to make a phone call than conduct a video conference call, inpatient phone visits required significant coordination among nursing staff before talking to patients directly. One provider noted that calling to check in with nurses rather than knocking on a door and entering the patient's room made it challenging to conduct early and frequent patient visits. Another mentioned that phone calls were not as efficient as direct visits to the inpatient hospital units because of the lack of direct, real-time interactions.

Video. Video visits were helpful in providing another means for communicating with patients. Care providers were able to see their patients, and their patients were able to see them. Video visits did not have the same limitations that phone visits did. A psychologist mentioned 
always opting for videos when possible in order to be better able to read his patients' body language. He reported that video care was done a lot more frequently in the outpatient setting. On the inpatient side of care, however, video consultations were not as frequently used for a variety of reasons. One care provider reported only providing video care for inpatients about $25 \%$ of the time. The coordination required to set up video consultations with inpatients involved multiple staff in the hospital, either to set up the video calls or to assist patients who were unable to hold up the tablets on their own.

Many felt that a major challenge to using video visits was the technological requirements. Video visits seemed to be difficult for older patients who were unfamiliar with the required technology. About half of the patients seen by palliative care at the VA were reported to live in rural areas and many of them had difficulty accessing the necessary technology for video visits. Some patients expressed their lack of trust in video appointments, although for unclear reasons, and chose not to participate. One OHSU physician reported that video support throughout the hospital was not robust.

\section{In-team communication}

Once providers began working remotely, the palliative care teams at both OHSU and the VA transitioned to conducting daily morning rounds via telephone. Before the pandemic, both interprofessional teams met at their respective institutions every morning to discuss their patient census, coordinate among each other, and strategize plans for the day.

One VA provider noted that since working remotely, having more inclusive discussions about treatment plans as a group was easier. At the Portland VA, providers used a combination of phone, WebEx, and Microsoft Teams to host their virtual communications. Many providers at the 
Portland VA reported a very quick transition to working virtually, despite challenges adjusting to it at first. One staff member mentioned that all meetings that he had held pre-pandemic were able to be held virtually without much issue. In the outpatient clinic at the VA, providers had an open chat room with video conferencing that allowed ease of real-time collaboration among the interprofessional team. This helped the members of the interprofessional palliative care team at the VA remain actively engaged while working remotely.

Outside of scheduled meetings, one provider found that working virtually made it hard to find pockets of time to communicate with colleagues. Coordinating with other providers outside of rounds was especially challenging for some.

At both OHSU and the VA, specific guidelines dictated which members of the interprofessional team could be present for in-person visits. 5 out of the 9 care providers interviewed reported issues regarding coordinating care within the team. One provider reported that due to the lack of direct interactions, phone calls with colleagues were less efficient, and some said virtual communication diminished the quality of in-team communication.

\section{Mental and psychological impact}

Providing care while quarantining impacted morale negatively, for both healthcare professionals and patients. Concerns expressed were feeling a loss of group cohesion and team mentality, as well as increased psychological distress. Due to visitor restrictions, providers had an increased need to balance family concerns. Providers also lost family support in aiding communications between them and their patients. One chaplain reported that staff in the hospital also felt a lack of support, specifically nurses who had to work when case management, social work, palliative care, and chaplains were no longer in hospital. 
For patients, there was not an ideal route for receiving care during the pandemic. Two care providers reported patients not wanting to come to the hospital for care due to fear of the virus. Three care providers reported difficulty creating a sense of trust with patients via virtual platforms of care. Feelings of engagement were also difficult to foster over the phone or video. Patients can naturally also not be very forthcoming about how they feel. One psychologist reported difficulty being present in appointments when patients have never seen the care provider's face, on top of meeting virtually. Virtual meetings were also seen as burdensome to patients, who grew tired of having many appointments to attend with their care providers. Patients who did not understand the non-medical service aspect of palliative care also had trouble staying engaged in receiving care, especially outpatient care.

\section{Positives}

Despite the many challenges palliative care providers faced while working during the pandemic, there were some positive facets to be noted about the new way of providing care. It had been noted by multiple professionals that doing things virtually can increase flexibility and access to care in certain aspects. One provider found it easier to send out invitations to their colleagues for more inclusive discussions. Phones allowed chaplains to participate in rounds while not having to come on campus. OHSU chaplains were given cell phones that could forward the calls from their work phones during business hours. This made calling families much easier and allowed chaplains to identify themselves with their OHSU IDs provided on the phones. Communicating with patients virtually also provided flexibility in scheduling and access to patients for care providers at the VA. 
With the introduction of vaccines, all palliative care providers at the VA have been fully immunized. This allowed them to transition back to working in the hospital more. Their workflow so far included working in-person more at least with the inpatient side of care, which had been reported to help better their service.

\section{Gaps in Knowledge}

As the pandemic continues, it is important to identify areas of missing knowledge in order to better prepare for future health crises. The COVID-19 pandemic brought about an urgent need and increased use of telehealth as a modality to provide care. However, it is still unknown going forward how healthcare professionals will incorporate telehealth into their work.

Most care providers reported feeling uncertain about the effectiveness of their work and communication when done virtually. The complexities of palliative and end-of-life care deserve to be addressed in an intimate setting and with the appropriate methods of communication. Times of solemn silence or a pat on the hand can help to deepen a conversation with a patient and build further trust. However, these nonverbal techniques did not translate over to telephone or video visits. Treatments, palliative care management, pain control, and support of patients' quality of life are some of the aspects of care that currently occupy a blind spot in the ability to measure effective virtual palliative care. Not being able to assess patients in person also prevented care providers from understanding thoroughly how patients are doing, especially if they were not forthcoming during virtual visits. Modifying a patient's quality of life was also difficult with social distancing and visitor restrictions, and care providers were left to fill such void in social support. For care providers whose disciplines were not allowed to visit patients in person, they 
faced the difficulty of navigating difficult palliative care topics virtually that their interprofessional counterparts did not.

Interdisciplinary and in-team collaboration was often not the same when done over the phone or videoconference. Teaching was reported to be impacted for worse by the pandemic due to restrictions on who can come to campus.

The need for more congruence in protocols and expectations of employees in terms of their own safety remains. The protocols for patient visitation should be determined by the hospital and not by the palliative care team after interprofessional discussions.

\section{Discussion}

In March of 2020, the world was faced with having to change how we conducted business, including how we cared for patients. As a result, many activities had to move to a virtual platform. Certainly, within healthcare, we showed that telemedicine became an important tool to use to continue to provide adequate care to patients. In some cases, remote care was a viable option and safe alternative for providing quality palliative care during a pandemic. Remote meetings provided more flexibility to be more all-inclusive, especially for those providers who were already not going to be onsite. Yet, challenging pieces were doing phone or video visits with patients and families because of technological difficulties and impacted modes of communication by being remote.

\section{Pandemic Preparations}

Pandemic preparations were crucially important in allowing for a smooth transition from working onsite to working remotely. The Portland VA's rapid deployment of telemedicine was in 
part due to their proprietary network as well as care providers' history of using it before the pandemic. Adequate video capabilities and a running system that hosted video appointments ensured that healthcare professionals were able to meet with their patients easily and securely. Having a secure, centralized system for patient communication took away the extra burden of figuring out how best to reach out to their patients when providers were not able to see them in person.

Video visits with patients also required the proper hardware, such as tablets. Limited access to tablets within hospitals can narrow down care options for inpatients to only phone if providers are not able to come to the hospital. However, as described by many providers, the process for setting up and carrying out a video visit for inpatients can be energetically taxing. Video calls required not only that the patient was well enough to engage in a conversation where they have to be present and visible to another person, but also need the setup done by onsite staff. In some cases, patients required nurses to hold their tablets for them during the appointment. As vaccines continue rolling out and care providers begin to work more on campus, the need for a video visit might diminish due to its more cumbersome setup process than phone calls. However, for the time being, and in preparation for a potential future health crisis, video support within hospitals can be more robust in terms of hardware and availability, perhaps even utilizing hands-free devices to make calls more accessible to all patients.

\section{Communication with patients}

Providing care virtually as a new normal way of work had been a great learning curve. The challenge in implementing virtual palliative care lied in two main areas: technological preparedness and the ability to compensate for the lack of nonverbal communication. 
Technological difficulties were not exclusive to palliative care when providing telemedicine; however, it can be more commonly encountered within palliative care since much of the patients seen belong to an older population. Aside from expertise or familiarity with technology, access to the proper equipment and setting it up can also be a hindrance to care. As noted, the process of transitioning to virtual work and setting up remotely can be challenging for providers. With video care, patients were more likely to not have the required equipment.

In terms of communication, video care had fewer limitations than phone care. Allowing patients to see their providers took away some of the disconnect between them and can make care feel more personal and engaging. However, there was a great discrepancy in the number of video appointments with outpatients compared to inpatients. There are a few possible reasons why video care is more efficient for outpatients than inpatients. Outpatients are typically less ill and have fewer physical limitations that could prevent them from attending their video appointments. Outpatients are also not dependent on the finite hospital resources provided to them - such as tablets - required for video care. Despite the certain limitations that video appointments have, they still remain a tremendously helpful option for providers to maintain as much of the quality of communication with patients and families as possible while working remotely.

For inpatient care, many providers found it to be more useful to reach out to them via phone calls or visit them in person if protocols allow. The challenges involved with providing care over the phone were mostly related to the quality of communication when providers and patients are not able to see each other. Phone calls with inpatients can be highly variable as well, although this can in part be attributed to the state of health of palliative care inpatients, as there also had been reports on the sporadic history of communicating with them virtually. However, 
phone calls allowed for a lot more flexibility and accessibility. Phone calls required less time and coordination with other providers to carry out than video calls, and phones were much more available, whether inpatient or outpatient. Conversations over the phone were typically shorter, allowing providers to reach out to patients more frequently.

\section{Communication within the team}

Telework has also impacted the communication between providers within the palliative care team. Due to the disconnected nature of telework, extra effort had to be given by every member to stay engaged with their team. What has been shown to be helpful was having allotted times when most or all team members can meet to discuss and exchange interdisciplinary consultations with each other, such as morning rounds, or in the case of the VA, an open chat room for providers to hop on and off whenever they have the chance. A benefit of working virtually, however, was that it was much easier to send out invitations to other providers for more inclusive discussions.

\section{Psychological impact of telemedicine on patients and providers}

Keeping patients engaged in care and showing up to virtual appointments had been a challenge for providers, whether with video or phone care. The impersonal nature of virtual care compared to seeing patients in person can make patients feel disinterested, especially if they began receiving palliative care during the pandemic and have not had a relationship with their providers previously. Furthermore, patients can grow tired of having to attend multiple meetings to check in with different care providers. A possible way to make sure that patients are engaged in their care and show up to their appointments is to have a care coordinator. This can be 
someone whose responsibility is to remind patients of their appointments, help them get set up and have everything they need before meeting with their providers.

For providers, working at home or separated from their team members can take a great psychological toll. Not being able to be in the same space and exchange ideas as well as support can be distressing, especially during this time of heightened fear and confusion. By having allotted times where team members can meet virtually, as well as setting up a virtual space for them to convene when they have the chance, providers can bring more support and connectedness within the team.

\section{Conclusion}

Telemedicine gives providers certain flexibility in time and better access to patients in some cases. Phone calls can take less time to carry out than an in-person appointment and can be done with more frequency since it does not require the patient or the provider to travel much. When meetings and interdisciplinary discussions all take place virtually, providers are able to attend by simply calling in, allowing for more inclusive collaboration.

While the option to visit care providers via a virtual platform has existed before the pandemic, the need for social distancing has undoubtedly popularized telemedicine. Even with its challenges, providers were able to deliver telehealthcare to patients with a steady frequency and quality. It is hard to determine exactly where the future of telehealth will go after this pandemic. However, the continuation and expansion of telemedicine can open up the opportunity to serve certain niches within the patient population -- those who prefer to receive care remotely. For example, with patients who live rurally, have busy schedules, or are less comfortable with talking to care providers face to face, telemedicine can give them a space to receive quality 
healthcare that fits their lifestyle. This could even expand the outreach of providers and encourage patients in some cases to receive care more, as certain providers have experienced during this pandemic.

\section{Areas for future research}

As the pandemic unfolds, more questions will arise about how best to deliver care to patients. The transition from an in-person workflow to a remote platform happened abruptly and has only been in place for a little more than a year. Providers have begun exploring the effects of the pandemic on their ability to provide palliative care. In the future, it will be interesting to explore and understand how patients perceived their care during the pandemic, as this study highlighted inferences about the perception of patients.

\section{Limitations}

The limitations of this study include a small sample size of providers and the timeframe in which the interviews were conducted. While the nine providers from two hospitals provided a multidisciplinary picture and functioned in two different institutions, the limited size of respondents and their shared proximity may limit the applicability of this study on a national scale. The interviews were also conducted within the timeframe of May of 2020 to February of 2021. As the pandemic continues, more challenges will arise and more information will be available. Therefore, this study did not provide information about providing palliative care with COVID-19 in the rearview mirror, but rather a look at how providers work now as the pandemic continues to develop. 


\section{References}

Bernacki, R. E., \& Block, S. D. (2014). Communication About Serious Illness Care Goals. JAMA Internal Medicine, 174(12), 1994. https://doi.org/10.1001/jamainternmed.2014.5271

Crawford, G. B., \& Price, S. D. (2003). Team working: palliative care as a model of interdisciplinary practice. Medical Journal of Australia, 179(S6). https://doi.org/10.5694/j.1326-5377.2003.tb05575.x

Lovell, N., Maddocks, M., Etkind, S. N., Taylor, K., Carey, I., Vora, V., Marsh, L., Higginson, I. J., Prentice, W., Edmonds, P., \& Sleeman, K. E. (2020). Characteristics, Symptom Management, and Outcomes of 101 Patients With COVID-19 Referred for Hospital Palliative Care. Journal of Pain and Symptom Management, 60(1), e77-e81. https://doi.org/10.1016/j.jpainsymman.2020.04.015

OHSU Facts $\mid$ OHSU. (n.d.). Oregon Health \& Sciences University. Retrieved May 21, 2021, from https://www.ohsu.edu/about/ohsu-facts

Ting, R., Edmonds, P., Higginson, I. J., \& Sleeman, K. E. (2020). Palliative care for patients with severe covid-19. BMJ, m2710. https://doi.org/10.1136/bmj.m2710

US Department of Veterans Affairs, Veterans Health Administration. (n.d.). VA.gov | Veterans Affairs. US Department of Veteran Affairs. Retrieved May 21, 2021, from https://www.portland.va.gov/about/index.asp 
What Are Palliative Care and Hospice Care? (2021, May 21). National Institute on Aging. https://www.nia.nih.gov/health/what-are-palliative-care-and-hospice-care 